Bull. Korean Math. Soc. 50 (2013), No. 1, pp. 25-36

http://dx.doi.org/10.4134/BKMS.2013.50.1.025

\title{
NONEMPTY INTERSECTION THEOREMS \\ AND SYSTEM OF GENERALIZED VECTOR EQUILIBRIUM PROBLEMS IN $F C$-SPACES
}

\author{
Rong-Hua He And Hong-Xu Li
}

\begin{abstract}
By using some existence theorems of maximal elements for a family of set-valued mappings involving a better admissible set-valued mapping under noncompact setting of $F C$-spaces, we present some nonempty intersection theorems for a family $\left\{G_{i}\right\}_{i \in I}$ in product $F C$-spaces. Then, as applications, some new existence theorems of equilibrium for a system of generalized vector equilibrium problems are proved in product $F C$-spaces. Our results improve and generalize some recent results.
\end{abstract}

\section{Introduction and preliminaries}

The vector variational inequality problem (in short, VVIP) was first introduced by Giannessi [17] in finite dimensional Euclidean spaces. Since then, the $V V I P$ has been generalized and applied by many authors in various directions. Inspired and motivated by applications of the VVIP with set-valued mappings, various generalized vector variational inequality problem (in short, $G V V I P$ ) and generalized vector equilibrium problem (in short, $G V E P$ ) have become important developed directions of the classical $V V I P$ (see, for example, $[1,3,4,6,15,22]$ and the book edited by F. Giannessi [18], and the references therein).

Recently, Ding [12] introduced and studied a system of generalized vector equilibrium problems in the product space of $G$-convex spaces. Let $X$ be a topological space and $I$ be any index set. Let $\left\{D_{i}\right\}_{i \in I},\left\{E_{i}\right\}_{i \in I},\left\{Y_{i}\right\}_{i \in I}$ and $\left\{Z_{i}\right\}_{i \in I}$ be four families of topological spaces, and let $Y=\prod_{i \in I} Y_{i}$. For each $i \in I$, let $T_{i}: X \rightarrow 2^{D_{i}}, S_{i}: X \rightarrow 2^{E_{i}}, C_{i}: X \rightarrow 2^{Z_{i}}$ and $\eta_{i}: D_{i} \times E_{i} \times Y_{i} \rightarrow 2^{Z_{i}}$ be set-valued mappings. A system of generalized vector equilibrium problems

Received September 1, 2010; Revised March 19, 2012.

2010 Mathematics Subject Classification. 49J40, 49J53.

Key words and phrases. maximal element, nonempty intersection theorem, system of generalized vector equilibrium problems, product $F C$-space.

This work is supported by the National Natural Science Foundation of China (11171046), the National Natural Science Foundation of China (11071042) and the Scientific Research Foundation of CUIT under Grant (KYTZ201114). 
(in short, $S G V E P$ ) is to find $\hat{x} \in X$ such that for each $i \in I$,

$$
\forall y_{i} \in Y_{i}, \exists \hat{d}_{i} \in T_{i}(\hat{x}), \hat{e}_{i} \in S_{i}(\hat{x}) \text { such that } \eta_{i}\left(\hat{d}_{i}, \hat{e}_{i}, y_{i}\right) \not C_{i}(\hat{x})
$$

Motivated and inspired by the above research works, we shall present some nonempty intersection theorems for a family $\left\{G_{i}\right\}_{i \in I}$ in a product $F C$-spaces, which are generalizations of $G$-convex spaces, by applying an existence theorems of maximal elements obtained by He and Zhang [20]. As applications, several new existence theorems of equilibrium points for the $S G V E P(1)$ are established in product $F C$-space. Our results improve and generalize the corresponding results in $[2,12,14,19,23]$.

Let $X$ and $Y$ be two nonempty sets. We denote by $2^{Y}$ and $\langle X\rangle$ the family of all subsets of $Y$ and the family of all nonempty finite subsets of $X$, respectively. Let $\Delta_{n}$ denote the standard $n$-dimensional simplex with the vertices $\left\{e_{0}, \ldots, e_{n}\right\}$. If $J$ is a nonempty subset of $\{0,1, \ldots, n\}$, we shall denote by $\triangle_{J}$ the convex hull of the vertices $\left\{e_{j}: j \in J\right\}$. For topological spaces $X$ and $Y$, a subset $A$ of $X$ is said to be compactly open (resp., compactly closed) if for each nonempty compact subset $K$ of $X, A \cap K$ is open (resp., closed) in $K$. The compact closure of $A$ and the compact interior of $A$ (see [5]) are defined respectively by

$$
\begin{aligned}
\text { ccl } A & =\bigcap\{B \subset X: A \subset B \text { and } B \text { is compactly closed in } X\}, \\
\operatorname{cint} A & =\bigcup\{B \subset X: B \subset A \text { and } B \text { is compactly open in } X\} .
\end{aligned}
$$

A set-valued mapping $T: X \rightarrow 2^{Y}$ is said to be transfer compactly closed valued on $X$ (see [5]) if for each $x \in X$ and $y \notin T(x)$, there exists $x^{\prime} \in X$ such that $y \notin \operatorname{ccl} T\left(x^{\prime}\right)$. T is said to be transfer compactly open valued on $X$ if for each $x \in X$ and $y \in T(x)$, there exists $x^{\prime} \in X$ such that $y \in \operatorname{cint} T\left(x^{\prime}\right)$.

First, we recall the following concepts (see $[13,20])$.

Definition 1.1. $\left(X, \varphi_{N}\right)$ is said to be an $F C$-space if $X$ is a topological space and for each $N=\left\{x_{0}, \ldots, x_{n}\right\} \in\langle X\rangle$, where some elements in $N$ may be same, there exists a continuous mapping $\varphi_{N}: \Delta_{n} \rightarrow X$. A subset $D$ of $X$ is said to be an $F C$-subspace of $X$ if for each $N=\left\{x_{0}, \ldots, x_{n}\right\} \in\langle X\rangle$ and for each $\left\{x_{i_{0}}, \ldots, x_{i_{k}}\right\} \subset N \cap D, \varphi_{N}\left(\Delta_{k}\right) \subset D$, where $\Delta_{k}=c o\left(\left\{e_{i_{j}}: j=0, \ldots, k\right\}\right)$.

Definition 1.2. An $F C$-space $\left(X, \varphi_{N}\right)$ is said to be a $C F C$-space if for each $N \in\langle Y\rangle$, there exists a compact $F C$-subspace $L_{N}$ of $Y$ containing $N$.

Now, we give some lemmas which will be useful in the proof of our main results (see $[13,20])$.

Lemma 1.1. Let $I$ be any index set. For each $i \in I$, let $\left(Y_{i}, \varphi_{N_{i}}\right)$ be an $F C$ space, $Y=\prod_{i \in I} Y_{i}$ and $\varphi_{N}=\prod_{i \in I} \varphi_{N_{i}}$. Then $\left(Y, \varphi_{N}\right)$ is also an $F C$-space.

The following notion was introduced by He and Zhang [20]. Let $X$ be a topological space and $I$ be any index set. For each $i \in I$, let $\left(Y_{i}, \varphi_{N_{i}}\right)_{i \in I}$ be an $F C$-space and $Y=\prod_{i \in I} Y_{i}$ such that $\left(Y, \varphi_{N}\right)$ is an $F C$-space defined as in 
Lemma 1.1. Let $F \in \mathcal{B}(Y, X)$ and $A_{i}: X \rightarrow 2^{Y_{i}}, i \in I$ be set-valued mappings. For each $i \in I$,

(1) $A_{i}: X \rightarrow 2^{Y_{i}}$ is said to be a generalized $G_{\mathcal{B}^{-} \text {-mapping if }}$

(a) for each $N=\left\{y_{0}, \ldots, y_{n}\right\} \in\langle Y\rangle$ and $\left\{y_{i_{0}}, \ldots, y_{i_{k}}\right\} \subset N$,

$$
F\left(\varphi_{N}\left(\Delta_{k}\right)\right) \bigcap\left(\bigcap_{j=0}^{k} \operatorname{cint} A_{i}^{-1}\left(\pi_{i}\left(y_{i_{j}}\right)\right)\right)=\emptyset
$$

where $\pi_{i}$ is the projection of $Y$ onto $Y_{i}$ and $\Delta_{k}=c o\left(\left\{e_{i_{j}}: j=\right.\right.$ $0, \ldots, k\})$;

(b) $A_{i}^{-1}\left(y_{i}\right)=\left\{x \in X: y_{i} \in A_{i}(x)\right\}$ is transfer compactly open in $Y_{i}$ for each $y_{i} \in Y_{i}$.

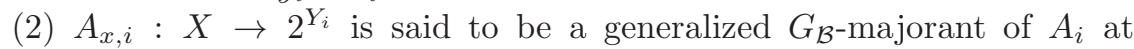
$x \in X$ if $A_{x, i}$ is a generalized $G_{\mathcal{B}}$-mapping and there exists an open neighborhood $N(x)$ of $x$ in $X$ such that $A_{i}(z) \subset A_{x, i}(z)$ for all $z \in$ $N(x)$.

(3) $A_{i}$ is said to be a generalized $G_{\mathcal{B}}$-majorized if for each $x \in X$ with $A_{i}(x) \neq \emptyset$, there exists a generalized $G_{\mathcal{B}}$-majorant $A_{x, i}$ of $A_{i}$ at $x$, and for any $N \in\left\langle\left\{x \in X: A_{i}(x) \neq \emptyset\right\}\right\rangle$, the mapping $\bigcap_{x \in N} A_{x, i}^{-1}$ is transfer compactly open in $Y_{i}$.

(4) $\left\{A_{i}\right\}_{i \in I}$ is said to be a family of generalized $G_{\mathcal{B}}$-mappings (resp., $G_{\mathcal{B}^{-}}$ majorant mappings) if for each $i \in I, A_{i}: X \rightarrow 2^{Y_{i}}$ is a generalized $G_{\mathcal{B}}$-mapping (resp., $G_{\mathcal{B}}$-majorant mapping).

Lemma 1.2. Let $X$ be a topological space, $K$ be a nonempty compact subset of $X$ and $I$ be any index set. For each $i \in I$, let $\left(Y_{i}, \varphi_{N_{i}}\right)$ be an $F C$-space and $Y=\prod_{i \in I} Y_{i}$ such that $\left(Y, \varphi_{N}\right)$ is an $F C$-space defined as in Lemma 1.1. Let $F \in \mathcal{B}(Y, X)$ and for each $i \in I, A_{i}: X \rightarrow 2^{Y_{i}}$ be a generalized $G_{\mathcal{B}-\text { mapping }}$ such that the following condition holds:

(P) For each $i \in I$ and $N_{i} \in\left\langle Y_{i}\right\rangle$, there exists a compact $F C$-subspace $L_{N_{i}}$ of $Y_{i}$ containing $N_{i}$ and for each $x \in X \backslash K$, there exists $i \in I$ satisfying $L_{N_{i}} \cap \operatorname{cint} A_{i}(x) \neq \emptyset$.

Then there exists $\hat{x} \in K$ such that $A_{i}(\hat{x})=\emptyset$ for each $i \in I$.

Lemma 1.3. Let $X$ be a topological space, and let $I$ be any index set. For each $i \in I$, let $\left(Y_{i}, \varphi_{N_{i}}\right)$ be an $C F C$-space, and let $Y=\prod_{i \in I} Y_{i}$. Let $F \in \mathcal{B}(Y, X)$ be a compact mapping such that the following two conditions hold for each $i \in I$ :

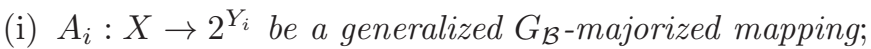

(ii) $\bigcup_{i \in I}\left\{x \in X: A_{i}(x) \neq \emptyset\right\}=\bigcup_{i \in I} \operatorname{cint}\left\{x \in X: A_{i}(x) \neq \emptyset\right\}$.

Then there exists $\hat{x} \in X$ such that $A_{i}(\hat{x})=\emptyset$ for each $i \in I$. 


\section{Nonempty intersection theorems}

Definition 2.1. Let $D, E, Y$ and $Z$ be nonempty sets and $X$ be a topological space. Let $F: D \times E \times Y \rightarrow 2^{Z}$ and $C: X \rightarrow 2^{Z}$ be two set-valued mappings. $F(d, e, y)$ is said to be transfer compactly upper semicontinuous in $x$ with respect to $C$ if for any nonempty compact subset $K$ of $X$ and any $x \in K$, $\{(d, e, y) \in D \times E \times Y: F(d, e, y) \subseteq C(x)\} \neq \emptyset$ implies that there exist a relatively open neighborhood $N(x)$ of $x$ in $K$ and $\left(d^{\prime}, e^{\prime}, y^{\prime}\right) \in D \times E \times Y$ such that $F\left(d^{\prime}, e^{\prime}, y^{\prime}\right) \subseteq C(z)$ for all $z \in N(x)$.

We first give the following nonempty intersection theorem which is in fact equivalent to Lemma 1.2.

Theorem 2.1. Let $X$ be a topological space, $K$ be a nonempty compact subset of $X$ and $I$ be any index set. For each $i \in I$, let $\left(Y_{i}, \varphi_{N_{i}}\right)$ be an $F C$-space and $Y=\prod_{i \in I} Y_{i}$ such that $\left(Y, \varphi_{N}\right)$ is an $F C$-space defined as in Lemma 1.1. Let $F \in \mathcal{B}(Y, X)$ and $G_{i}: Y_{i} \rightarrow 2^{X}$ be such that the following conditions hold:

(i) For each $y_{i} \in Y_{i}, G_{i}\left(y_{i}\right)$ is transfer compactly closed in $Y_{i}$.

(ii) For each $N=\left\{y_{0}, \ldots, y_{n}\right\} \in\langle Y\rangle$ and $\left\{y_{r_{0}}, \ldots, y_{r_{k}}\right\} \subset N$,

$$
F\left(\varphi_{N}\left(\Delta_{k}\right)\right) \bigcap\left(\bigcap_{j=0}^{k} \operatorname{cint}\left(X \backslash G_{i}\left(\pi_{i}\left(y_{r_{j}}\right)\right)\right)\right)=\emptyset \text {, }
$$

where $\pi_{i}$ is the projection of $Y$ onto $Y_{i}$ and $\Delta_{k}=\operatorname{co}\left(\left\{e_{r_{j}}: j=\right.\right.$ $0, \ldots, k\})$.

(iii) For each $i \in I$ and $N_{i} \in\left\langle Y_{i}\right\rangle$, there exists a compact $F C$-subspace $L_{N_{i}}$ of $Y_{i}$ containing $N_{i}$ and for each $x \in X \backslash K$, there exists $i \in I$ satisfying $L_{N_{i}} \cap \operatorname{cint}\left(Y_{i} \backslash G_{i}^{-1}(x)\right) \neq \emptyset$.

Then we have

$$
K \bigcap\left(\bigcap_{i \in I} \bigcap_{y_{i} \in Y_{i}} G_{i}\left(y_{i}\right)\right) \neq \emptyset .
$$

Proof. Lemma 1.2 $\Rightarrow$ Theorem 2.1. For each $i \in I$, define a set-valued mapping $A_{i}: X \rightarrow 2^{Y_{i}}$ by

$$
A_{i}(x)=Y_{i} \backslash G_{i}^{-1}(x), \quad \forall x \in X .
$$

Then for each $y_{i} \in Y_{i}$, we have

$$
\begin{aligned}
A_{i}^{-1}\left(y_{i}\right) & =\left\{x \in X: y_{i} \in A_{i}(x)\right\}=\left\{x \in X: y_{i} \in Y_{i} \backslash G_{i}^{-1}(x)\right\} \\
& =\left\{x \in X: x \notin G_{i}\left(y_{i}\right)\right\}=X \backslash G_{i}\left(y_{i}\right) .
\end{aligned}
$$

Hence $A_{i}^{-1}\left(y_{i}\right)$ is transfer compactly open in $X$ by condition (i). The condition (ii) of Theorem 2.1 implies that for each $N=\left\{y_{0}, \ldots, y_{n}\right\} \in\langle Y\rangle$ and $\left\{y_{r_{0}}, \ldots, y_{r_{k}}\right\} \subset N$,

$$
F\left(\varphi_{N}\left(\Delta_{k}\right)\right) \bigcap\left(\bigcap_{j=0}^{k} \operatorname{cint} A_{i}^{-1}\left(\pi_{i}\left(y_{r_{j}}\right)\right)\right)=\emptyset,
$$


where $\pi_{i}$ is the projection of $Y$ onto $Y_{i}$ and $\Delta_{k}=c o\left(\left\{e_{r_{j}}: j=0, \ldots, k\right\}\right)$. So for each $i \in I, A_{i}: X \rightarrow 2^{Y_{i}}$ is a generalized $G_{\mathcal{B}}$-mapping. The condition (iii) of Theorem 2.1 implies that the condition (P) of Lemma 1.2 holds. Then by Lemma 1.2, there exists $\hat{x} \in K$ such that $A_{i}(\hat{x})=\emptyset$ for each $i \in I$. Therefore, we have $A_{i}(\hat{x})=Y_{i} \backslash G_{i}^{-1}(\hat{x})=\emptyset$, and then $Y_{i}=G_{i}^{-1}(\hat{x})$ for $i \in I$. This implies that

$$
\hat{x} \in \bigcap_{i \in I} \bigcap_{y_{i} \in Y_{i}} G_{i}\left(y_{i}\right) .
$$

Hence we obtain

$$
K \bigcap\left(\bigcap_{i \in I} \bigcap_{y_{i} \in Y_{i}} G_{i}\left(y_{i}\right)\right) \neq \emptyset .
$$

Theorem 2.1 $\Rightarrow$ Lemma 1.2. For each $i \in I$, define a set-valued mapping $G_{i}: Y_{i} \rightarrow 2^{X}$ by

$$
G_{i}\left(y_{i}\right)=X \backslash A_{i}^{-1}\left(y_{i}\right), \forall y_{i} \in Y_{i} .
$$

Since for each $i \in I, A_{i}: X \rightarrow 2^{Y_{i}}$ is a generalized $G_{\mathcal{B}}$-mapping, conditions (i) and (ii) of Theorem 2.1 are satisfied. Condition (P) of Lemma 1.2 implies that condition (iii) of Theorem 2.1 holds. Consequently, all conditions of Theorem 2.1 are satisfied, and then we have

$$
K \bigcap\left(\bigcap_{i \in I} \bigcap_{y_{i} \in Y_{i}} G_{i}\left(y_{i}\right)\right) \neq \emptyset .
$$

Taking any $\hat{x} \in K \bigcap\left(\bigcap_{i \in I} \bigcap_{y_{i} \in Y_{i}} G_{i}\left(y_{i}\right)\right) \neq \emptyset$, we have $\hat{x} \in K$ and

$$
\hat{x} \in G_{i}\left(y_{i}\right)=X \backslash A_{i}^{-1}\left(y_{i}\right) \quad \text { for } y_{i} \in Y_{i}, i \in I,
$$

which implies

$$
y_{i} \notin A_{i}(\hat{x}) \quad \text { for } y_{i} \in Y_{i}, i \in I .
$$

So $A_{i}(\hat{x})=\emptyset$ for all $i \in I$. This completes the proof.

Remark 2.1. Since Theorem 2.1 is an equivalent form of Lemma 1.2, Theorem 2.1 generalizes Theorem 2 and Theorem 4 of Park and Kim [23]. Moreover, Theorem 2.1 improves and generalizes Theorem 2.1 of Ding [12] in the following ways: (a) The setting spaces are generalized from " $G$-convex spaces" to "FC-spaces" without linear structure; (b) The assumption for mapping $G_{i}$ is generalized from "compactly closed" to "transfer compactly closed"; (c) Conditions (ii) and (iii) of Theorem 2.1 are weaker than conditions (ii) and (iii) of Theorem 2.1 in [12].

Especially, if $X=Y$ in Theorem 2.1, we have the following result, which improves Theorem 3.2 in Ding [14].

Corollary 2.1. Let $I$ be any index set. For each $i \in I$, let $\left(X_{i}, \varphi_{N_{i}}\right)$ be an $F C$-space and $X=\prod_{i \in I} X_{i}$ such that $\left(X, \varphi_{N}\right)$ is an FC-space defined as in 
Lemma 1.1. Let $K$ be a nonempty compact subset of $X$ and $G_{i}: X_{i} \rightarrow 2^{X}$ be such that the following conditions hold:

(i) For each $x_{i} \in X_{i}, G_{i}\left(x_{i}\right)$ is transfer compactly closed in $X_{i}$.

(ii) For each $N=\left\{x_{0}, \ldots, x_{n}\right\} \in\langle X\rangle$ and $\left\{x_{r_{0}}, \ldots, x_{r_{k}}\right\} \subset N$,

$$
\varphi_{N}\left(\Delta_{k}\right) \bigcap\left(\bigcap_{j=0}^{k} \operatorname{cint}\left(X \backslash G_{i}\left(\pi_{i}\left(x_{r_{j}}\right)\right)\right)\right)=\emptyset \text {, }
$$

where $\pi_{i}$ is the projection of $X$ onto $X_{i}$ and $\Delta_{k}=\operatorname{co}\left(\left\{e_{r_{j}}: j=\right.\right.$ $0, \ldots, k\})$.

(iii) For each $i \in I$ and $N_{i} \in\left\langle X_{i}\right\rangle$, there exists a compact $F C$-subspace $L_{N_{i}}$ of $X_{i}$ containing $N_{i}$ and for each $x \in X \backslash K$, there exists $i \in I$ satisfying $L_{N_{i}} \cap \operatorname{cint}\left(X_{i} \backslash G_{i}^{-1}(x)\right) \neq \emptyset$.

Then we have

$$
K \bigcap\left(\bigcap_{i \in I} \bigcap_{x_{i} \in X_{i}} G_{i}\left(x_{i}\right)\right) \neq \emptyset .
$$

Proof. For each $i \in I$, let $Y_{i}=X_{i}, X=Y=\prod_{i \in I} X_{i}$ and $F$ be the identity mapping on $X$. Then all the conditions of Theorem 2.1 are satisfied and the proof is completed.

If $X_{i}$ in Corollary 2.1 is a compact $F C$-space for each $I$, we have the following result with simpler form.

Corollary 2.2. Let $I$ be any index set. For each $i \in I$, let $\left(X_{i}, \varphi_{N_{i}}\right)$ be a compact $F C$-space and $X=\prod_{i \in I} X_{i}$ such that $\left(X, \varphi_{N}\right)$ is an $F C$-space defined as in Lemma 1.1. Let $G_{i}: X_{i} \rightarrow 2^{X}$ be such that the following conditions hold for each $i \in I$ :

(i) for each $x_{i} \in X_{i}, G_{i}\left(x_{i}\right)$ is transfer compactly closed in $X_{i}$;

(ii) for each $N=\left\{x_{0}, \ldots, x_{n}\right\} \in\langle X\rangle$ and $\left\{x_{r_{0}}, \ldots, x_{r_{k}}\right\} \subset N$, $\varphi_{N}\left(\Delta_{k}\right) \bigcap\left(\bigcap_{j=0}^{k} \operatorname{cint}\left(X \backslash G_{i}\left(\pi_{i}\left(x_{r_{j}}\right)\right)\right)\right)=\emptyset$, where $\pi_{i}$ is the projection of $X$ onto $X_{i}$ and $\Delta_{k}=c o\left(\left\{e_{r_{j}}: j=0, \ldots, k\right\}\right)$.

Then we have

$$
\bigcap_{i \in I} \bigcap_{x_{i} \in X_{i}} G_{i}\left(x_{i}\right) \neq \emptyset
$$

Proof. For each $i \in I$ and $N_{i} \in\left\langle X_{i}\right\rangle$, let $L_{N_{i}}=X_{i}$ and let $K=\prod_{i \in I} X_{i}$. Then $K=X$ is compact. Clearly the condition (iii) of Corollary 2.1 is satisfied trivially. The conclusion of Corollary 2.2 holds from Corollary 2.1.

By Corollary 2.1, we also have the following result, which improves Theorem 3.3 of Ding [14].

Corollary 2.3. Let $I$ be any index set. For each $i \in I$, let $\left(X_{i}, \varphi_{N_{i}}\right)$ be an $F C$-space and $X=\prod_{i \in I} X_{i}$ such that $\left(X, \varphi_{N}\right)$ is an $F C$-space defined as in 
Lemma 1.1, and let $K$ be a nonempty compact subset of $X$. Let $G_{i}: X_{i} \rightarrow 2^{X}$ be such that the following conditions hold for each $i \in I$ :

(i) For each $x_{i} \in X_{i}, G_{i}\left(x_{i}\right)$ is transfer compactly closed in $X_{i}$.

(ii) For each $x \in X, X_{i} \backslash G_{i}^{-1}(x)$ is an FC-subspace of $X_{i}$.

(iii) For each $x \in X, \pi_{i}(x) \in G_{i}^{-1}(x)$.

(iv) For each $i \in I$ and $N_{i} \in\left\langle X_{i}\right\rangle$, there exists a compact $F C$-subspace $L_{N_{i}}$ of $X_{i}$ containing $N_{i}$ and for each $x \in X \backslash K$, there exists $i \in I$ satisfying $L_{N_{i}} \cap \operatorname{cint}\left(X_{i} \backslash G_{i}^{-1}(x)\right) \neq \emptyset$.

Then we have

$$
K \bigcap\left(\bigcap_{i \in I} \bigcap_{x_{i} \in X_{i}} G_{i}\left(x_{i}\right)\right) \neq \emptyset .
$$

Proof. We first show that conditions (ii) and (iii) imply conditions (ii) of Corollary 2.1 holds. If it is false, then there exist $N=\left\{x_{0}, \ldots, x_{n}\right\} \in\langle X\rangle$ and $M=\left\{x_{r_{0}}, \ldots, x_{r_{k}}\right\} \subset N$ such that

$$
\varphi_{N}\left(\Delta_{k}\right) \bigcap\left(\bigcap_{j=0}^{k} \operatorname{cint}\left(X \backslash G_{i}\left(\pi_{i}\left(x_{r_{j}}\right)\right)\right)\right) \neq \emptyset .
$$

Hence there exists $\hat{x} \in \varphi_{N}\left(\Delta_{k}\right)=\prod_{i \in I} \varphi_{N_{i}}\left(\Delta_{k}\right)$ where $N_{i}=\pi_{i}(N)$ such that $\hat{x} \notin G_{i}\left(\pi_{i}(x)\right)$ for all $x \in M$. So we have $\pi_{i}(M) \subseteq X_{i} \backslash G_{i}^{-1}(\hat{x})$. Since $X_{i} \backslash G_{i}^{-1}(\hat{x})$ is an FC-subspace of $X_{i}$ by (ii), we have $\pi_{i}\left(\varphi_{N}\left(\Delta_{k}\right)\right) \subset X_{i} \backslash$ $G_{i}^{-1}(\hat{x})$. So $\pi_{i}(\hat{x}) \in X_{i} \backslash G_{i}^{-1}(\hat{x})$ and then $\pi_{i}(\hat{x}) \notin G_{i}^{-1}(\hat{x})$, which contradicts the condition (iii). This show that the conditions (ii) and (iii) imply the condition (ii) of Corollary 2.1 holds. Corollary 2.3 follows from Corollary 2.1.

As a direct consequence of Corollary 2.3, we have the following result, which generalizes and improves Theorem 2.1 of Guillerme [19].

Corollary 2.4. Let $I$ be any index set. For each $i \in I$, let $\left(X_{i}, \varphi_{N_{i}}\right)$ be a compact $F C$-space and $X=\prod_{i \in I} X_{i}$ such that $\left(X, \varphi_{N}\right)$ is an FC-space defined as in Lemma 1.1. Let $G_{i}: X_{i} \rightarrow 2^{X}$ be such that the following conditions hold for each $i \in I$ :

(iii) For each $x_{i} \in X_{i}, G_{i}\left(x_{i}\right)$ is transfer compactly closed in $X_{i}$.

(iii) For each $x \in X, X_{i} \backslash G_{i}^{-1}(x)$ is an FC-subspace of $X_{i}$.

(iii) For each $x \in X, \pi_{i}(x) \in G_{i}^{-1}(x)$.

Then we have

$$
\bigcap_{i \in I} \bigcap_{x_{i} \in X_{i}} G_{i}\left(x_{i}\right) \neq \emptyset .
$$

\section{System of generalized vector equilibrium problems}

By using Theorem 2.1, we establish some new equilibrium existence results for $S G V E P(1)$ in this section. 
Theorem 3.1. Let $X$ be a topological space, $K$ be a nonempty compact subset of $X, I$ be any index set, $\left(Y_{i}, \varphi_{N_{i}}\right)$ be a family of FC-spaces, $\left\{D_{i}\right\}_{i \in I},\left\{E_{i}\right\}_{i \in I}$ and $\left\{Z_{i}\right\}_{i \in I}$ be the families of topological spaces, and $Y=\prod_{i \in I} Y_{i}$ such that $\left(Y, \varphi_{N}\right)$ is an $F C$-space defined as in Lemma 1.1. Let $F \in \mathcal{B}(Y, X)$, and for each $i \in I, T_{i}: X \rightarrow 2^{D_{i}}, S_{i}: X \rightarrow 2^{E_{i}}, C_{i}: X \rightarrow 2^{Z_{i}}$ and $\eta_{i}: D_{i} \times E_{i} \times Y_{i} \rightarrow$ $2^{Z_{i}}$ be set valued mappings such that the following conditions hold for each $i \in I$ :

(i) $\eta_{i}\left(d_{i}, e_{i}, y_{i}\right)$ is transfer compactly upper semicontinuous in $x$ with respect to $C_{i}$.

(ii) For each $N=\left\{y_{0}, \ldots, y_{n}\right\} \in\langle Y\rangle$ and $N_{1}=\left\{y_{r_{0}}, \ldots, y_{r_{k}}\right\} \subset N$, and for each $x \in F\left(\varphi_{N}\left(\Delta_{k}\right)\right)$, there exists $d_{i} \in T_{i}(x), e_{i} \in S_{i}(x)$ and $y \in N_{1}$ such that $\eta_{i}\left(d_{i}, e_{i}, \pi_{i}(y)\right) \nsubseteq C_{i}(x)$.

(iii) For each $N_{i} \in\left\langle Y_{i}\right\rangle$, there exists a compact $F C$-subspace $L_{N_{i}}$ of $Y_{i}$ containing $N_{i}$, and for each $x \in X \backslash K$, there exist $i \in I$ and $y_{i} \in L_{N_{i}}$ satisfying

$y_{i} \notin \operatorname{ccl}\left\{u_{i} \in Y_{i}: \exists d_{i} \in T_{i}(x), e_{i} \in S_{i}(x)\right.$ such that $\left.\eta_{i}\left(d_{i}, e_{i}, u_{i}\right) \nsubseteq C_{i}(x)\right\}$.

Then there exists $\hat{x} \in K$ satisfying that, for each $i \in I$ and $y_{i} \in Y_{i}$, there exist $\hat{d}_{i} \in T_{i}(\hat{x})$ and $\hat{e_{i}} \in S_{i}(\hat{x})$ such that $\eta_{i}\left(\hat{d}_{i}, \hat{e}_{i}, y_{i}\right) \nsubseteq C_{i}(\hat{x})$.

Proof. For each $i \in I$, defined set-valued mappings $T_{i}, H_{i}: Y_{i} \rightarrow 2^{X}$ by

$$
\begin{aligned}
T_{i}\left(y_{i}\right) & =\left\{x \in X: \exists d_{i} \in T_{i}(x) \text { and } e_{i} \in S_{i}(x) \text { such that } \eta_{i}\left(d_{i}, e_{i}, y_{i}\right) \nsubseteq C_{i}(x)\right\}, \\
H_{i}\left(y_{i}\right) & =X \backslash T_{i}\left(y_{i}\right) \\
& =\left\{x \in X: \exists d_{i} \in T_{i}(x) \text { and } e_{i} \in S_{i}(x) \text { such that } \eta_{i}\left(d_{i}, e_{i}, y_{i}\right) \subseteq C_{i}(x)\right\} .
\end{aligned}
$$

Notice that for each $i \in I, \eta_{i}\left(d_{i}, e_{i}, y_{i}\right)$ is transfer compactly upper semicontinuous in $x$ with respect to $C_{i}$. Then for any nonempty compact subset $K$ of $X$, if $x \in H_{i}\left(y_{i}\right) \bigcap K$, we have $x \in K$ and there exist $d_{i} \in T_{i}(x)$ and $e_{i} \in S_{i}(x)$ such that $\eta_{i}\left(d_{i}, e_{i}, y_{i}\right) \subseteq C_{i}(x)$, i.e.,

$$
\left\{\left(d_{i}, e_{i}, y_{i}\right) \in D_{i} \times E_{i} \times Y_{i}: \eta_{i}\left(d_{i}, e_{i}, y_{i}\right) \subseteq C_{i}(x)\right\} \neq \emptyset .
$$

By condition (i), there exists a relatively open neighborhood $N(x)$ of $x$ in $K$ and $\left(d_{i}^{\prime}, e_{i}^{\prime}, y_{i}^{\prime}\right) \in D_{i} \times E_{i} \times Y_{i}$ such that $\eta_{i}\left(d_{i}^{\prime}, e_{i}^{\prime}, y_{i}^{\prime}\right) \subseteq C_{i}(z)$ for all $z \in N(x)$. Then we have

$$
\begin{aligned}
x \in N(x) & \subseteq \operatorname{int}_{K}\left(K \bigcap\left\{z \in X: \eta_{i}\left(d_{i}^{\prime}, e_{i}^{\prime}, y_{i}^{\prime}\right) \subseteq C_{i}(z)\right\}\right) \\
& =\operatorname{int}_{K}\left(H_{i}\left(y_{i}^{\prime}\right) \bigcap K\right)=\operatorname{cint}\left(H_{i}\left(y_{i}^{\prime}\right)\right) \bigcap K .
\end{aligned}
$$

This implies that $H_{i}$ is transfer compactly open in $Y_{i}$. Thus for each $i \in I$ and $y_{i} \in Y_{i}, T_{i}\left(y_{i}\right)$ is transfer compactly closed in $Y_{i}$. So condition (i) of Theorem 2.1 is satisfied. 
From the condition (ii) it follows that for each $N=\left\{y_{0}, \ldots, y_{n}\right\} \in\langle Y\rangle$ and $N_{1}=\left\{y_{r_{0}}, \ldots, y_{r_{k}}\right\} \subset N$, we have

$$
F\left(\varphi_{N}\left(\Delta_{k}\right)\right) \subseteq \bigcup_{y \in N_{1}} T_{i}\left(\pi_{i}(y)\right) \subset \bigcup_{y \in N_{1}} c c l T_{i}\left(\pi_{i}(y)\right),
$$

and then

$$
\begin{aligned}
& F\left(\varphi_{N}\left(\Delta_{k}\right)\right) \bigcap\left(\bigcap_{y \in N_{1}}\left(X \backslash c c l T_{i}\left(\pi_{i}(y)\right)\right)\right) \\
& =F\left(\varphi_{N}\left(\Delta_{k}\right)\right) \bigcap\left(\bigcap_{y \in N_{1}} \operatorname{cint}\left(X \backslash T_{i}\left(\pi_{i}(y)\right)\right)\right)=\emptyset .
\end{aligned}
$$

This yields that condition (ii) of Theorem 2.1 is satisfied.

For each $i \in I$ and $x \in X$, we have

$$
T_{i}^{-1}(x)=\left\{y_{i} \in Y_{i}: \exists d_{i} \in T_{i}(x), e_{i} \in S_{i}(x) \text { such that } \eta_{i}\left(d_{i}, e_{i}, y_{i}\right) \not C_{i}(x)\right\},
$$

and hence

$$
Y_{i} \backslash T_{i}^{-1}(x)=\left\{y_{i} \in Y_{i}: \eta_{i}\left(d_{i}, e_{i}, y_{i}\right) \subseteq C_{i}(x), \forall d_{i} \in T_{i}(x), e_{i} \in S_{i}(x)\right\} .
$$

By condition (iii), for each $N_{i} \in\left\langle Y_{i}\right\rangle$, there exists a compact $F C$-subspace $L_{N_{i}}$ of $Y_{i}$ containing $N_{i}$, and for each $x \in X \backslash K$, there exist $i \in I$ and $y_{i} \in L_{N_{i}}$ satisfying

So we have

$$
y_{i} \in Y_{i} \backslash \operatorname{ccl} T_{i}^{-1}(x)=\operatorname{cint}\left(Y_{i} \backslash T_{i}^{-1}(x)\right) .
$$

$$
L_{N_{i}} \bigcap \operatorname{cint}\left(Y_{i} \backslash T_{i}^{-1}(x)\right) \neq \emptyset .
$$

Then condition (iii) of Theorem 2.1 is satisfied. Now by Theorem 2.1 we have

$$
K \bigcap\left(\bigcap_{i \in I} \bigcap_{y_{i} \in Y_{i}} T_{i}\left(y_{i}\right)\right) \neq \emptyset .
$$

Taking any $\hat{x} \in K \bigcap\left(\bigcap_{i \in I} \bigcap_{y_{i} \in Y_{i}} T_{i}\left(y_{i}\right)\right)$, we obtain that $\hat{x} \in K$, and for each $i \in I$ and $y_{i} \in Y_{i}$ there exist $\hat{d}_{i} \in T_{i}(\hat{x})$ and $\hat{e}_{i} \in S_{i}(\hat{x})$ such that $\eta_{i}\left(\hat{d}_{i}, \hat{e}_{i}, y_{i}\right) \not \subset$ $C_{i}(\hat{x})$. This means that $\hat{x}$ is an equilibrium point of the $\operatorname{SGVEP}(1)$. The proof is complete.

Corollary 3.1. Let $I$ be any index set, $\left(X_{i}, \varphi_{N_{i}^{\prime}}\right)_{i \in I}$ and $\left(Y_{i}, \varphi_{N_{i}}\right)_{i \in I}$ be two families of $F C$-spaces. Let $\left\{Z_{i}\right\}_{i \in I}$ be a family of topological spaces and $X=$ $\prod_{i \in I} X_{i}, Y=\prod_{i \in I} Y_{i}$ such that $\left(X, \varphi_{N^{\prime}}\right),\left(Y, \varphi_{N}\right)$ are $F C$-spaces defined as in Lemma 1.1. Let $F: Y \rightarrow X$ be a continuous single-valued mapping and for each $i \in I, C_{i}: X \rightarrow 2^{Z_{i}}, f_{i}: X \times Y_{i} \rightarrow 2^{Z_{i}}$ be set valued mappings such that the following conditions holds:

(i) For each $i \in I, f_{i}\left(x, y_{i}\right)$ is transfer compactly upper semicontinuous in $x$ with respect to $C_{i}$. 
(ii) For each $N=\left\{y_{0}, \ldots, y_{n}\right\} \in\langle Y\rangle$ and $N_{1}=\left\{y_{r_{0}}, \ldots, y_{r_{k}}\right\} \subset N$, and for each $x \in F\left(\varphi_{N}\left(\Delta_{k}\right)\right)$, there exists $y \in N_{1}$ such that $f_{i}\left(x, \pi_{i}(y)\right) \nsubseteq$ $C_{i}(x)$.

(iii) There exists a nonempty compact subset $K_{i}$ of $X_{i}$. Let $K=\prod_{i \in I} K_{i}$. For each $N_{i} \in\left\langle Y_{i}\right\rangle$, there exists a compact $F C$-subspace $L_{N_{i}}$ of $Y_{i}$ containing $N_{i}$, and for each $x \in X \backslash K$, there exist $i \in I$ and $y_{i} \in L_{N_{i}}$ satisfying

$$
y_{i} \notin \operatorname{ccl}\left\{u_{i} \in Y_{i}: f_{i}\left(x, u_{i}\right) \nsubseteq C_{i}(x)\right\} .
$$

Then there exists $\hat{x} \in K$ such that for each $i \in I$ and $y_{i} \in Y_{i}$ we have $f_{i}\left(\hat{x}, y_{i}\right) \nsubseteq$ $C_{i}(\hat{x})$.

Proof. Clearly $K=\prod_{i \in I} K_{i}$ is a nonempty compact subset of $X$. For each $i \in I$, let $D_{i}=X_{i}$ and $E_{i}=X^{i}=\prod_{j \in I, j \neq i} X_{j}$. Write $x=\left(x_{i}, x^{i}\right)$ and $f_{i}(x, y i)=\eta_{i}\left(x_{i}, x^{i}, y i\right)$ for all $x \in X$ and $y_{i} \in Y_{i}$. Let $T_{i}(x)=\pi_{i}(x)=x_{i}$ and $S_{i}(x)=\pi^{i}(x)=x^{i}$ for each $i \in I$ and $x \in X$ where $\pi_{i}$ and $\pi^{i}$ are the projection mappings from $X$ onto $X_{i}$ and $X^{i}$, respectively. It is easy to check that all conditions of Theorem 3.1 are satisfied. By Theorem 3.1, there exists $\hat{x} \in K$ such that for each $i \in I$,

$$
f_{i}\left(\hat{x}, y_{i}\right)=\eta_{i}\left(\hat{x}_{i}, \hat{x}^{i}, y i\right) \nsubseteq C_{i}(\hat{x}), \forall y_{i} \in Y_{i} .
$$

This completes the proof.

Remark 3.1. (I) Theorem 3.1 generalizes Theorem 3.1 in [12] in several aspects: (a) The setting spaces are generalized from "family of $G$-convex spaces" to "family of $F C$-space" without linear structure; (b) Conditions (i), (ii) and (iii) of Theorem 3.1 in [12] are replaced by condition (i) of Theorem 3.1; (c) conditions (ii) and (iii) of Theorem 3.1 are weaker than conditions (iv) and (v) of Theorem 3.1 in [12].

(II) Corollary 3.1 generalizes Theorem 3.2 in [12] in the aspects similar as those of Theorem 3.1 to Theorem 3.1 in [12]. Moreover, Corollary 3.1 improves Theorem 2.1 and Theorem 2.2 in [2] from topological vector spaces to $F C$-space under weaker assumptions.

When $I$ be a singleton in Corollary 3.1, we obtain the following result.

Corollary 3.2. Let $I$ be any index set, $\left(X, \varphi_{N^{\prime}}\right)$ and $\left(Y, \varphi_{N}\right)$ be two $F C$ spaces and $Z$ be a topological space, $F: Y \rightarrow X$ be a continuous single-valued mapping, $C: X \rightarrow 2^{Z}$ and $f: X \times Y \rightarrow 2^{Z}$ be set-valued mappings such that

(i) For each $f(x, y)$ is transfer compactly upper semicontinuous in $x$ with respect to $C$.

(ii) For each $N=\left\{y_{0}, \ldots, y_{n}\right\} \in\langle Y\rangle, N_{1}=\left\{y_{r_{0}}, \ldots, y_{r_{k}}\right\} \subset N$ and $x \in$ $F\left(\varphi_{N}\left(\Delta_{k}\right)\right)$, there exists $y \in N_{1}$ such that $f(x, y) \nsubseteq C(x)$.

(iii) There exists a nonempty compact subset $K$ of $X$. For each $N \in\langle Y\rangle$, there exists a compact $F C$-subspace $L_{N}$ of $Y$ containing $N$, and for 
each $x \in X \backslash K$, there exists $y \in L_{N}$ satisfying

$$
y \notin \operatorname{ccl}\{u \in Y: f(x, u) \nsubseteq C(x)\} .
$$

Then there exists $\hat{x} \in X$ such that $f(\hat{x}, y) \nsubseteq C(\hat{x})$ for $y \in Y$.

\section{References}

[1] Q. H. Ansari, I. V. Konnov, and J.-C. Yao, On generalized vector equilibrium problems, Nonlinear Anal. 47 (2001), no. 1, 543-554.

[2] Q. H. Ansari, S. Schaible, and J. Y. Yao, System of vector equilibrium problems and its applications, J. Optim. Theory Appl. 107 (2000), no. 3, 547-557.

[3] Q. H. Ansari, A. H. Siddiqi, and S. Y. Wu, Existence and duality of generalized vector equilibrium problems, J. Math. Anal. Appl. 259 (2001), no. 1, 115-126.

[4] C. M. Chen, KKM property and fixed point theorems in metric spaces, J. Math. Anal. Appl. 323 (2006), no. 2, 1231-1237.

[5] C. M. Chen and T. H. Chang, Some results for the family $K K M(X, Y)$ and the Ф-mapping, J. Math. Anal. Appl. 329 (2007), no. 1, 92-101.

[6] C. M. Chen, T. H. Chang, and Y. P. Liao, Coincidence theorems, generalized variational inequality theorems and minimax inequality theorems for the $\Phi$-mapping on $G$-convex spaces, Fixed Point Theory Appl. 2007 (2007), Art. ID 78696, 13 pp.

[7] P. Deguire and M. Lassonde, Families sélectantes, Topol. Methods Nonlinear Anal. 5 (1995), no. 2, 261-269.

[8] P. Deguire, K. K. Tan, and X. Z. Yuan, The study of maximal elements, fixed points for $L_{\mathcal{S}}$-majorized mappings and their applications to minimax and variational inequalities in product topological spaces, Nonlinear Anal. 37 (1999), no. 7, 933-951.

[9] X. P. Ding, New $H-K K M$ theorems and their applications to geometric property, coincidence theorems, minimax inequality and maximal elements, Indian J. Pure Appl. Math. 26 (1995), no. 1, 1-19.

[10] — Fixed points, minimax inequalities and equilibria of noncompact abstract economies, Taiwanese J. Math. 2 (1998), no. 1, 25-55.

[11] Maximal elements for $G_{\mathcal{B}}$-majorized mappings in product $G$-convex spaces. II, Appl. Math. Mech. 24 (2003), no. 9, 1017-1024.

[12] _ Nonempty intersection theorems and system of generalized vector equilibrium problems in product $G$-convex spaces, Appl. Math. Mech. 25 (2004), no. 6, 618-626.

[13] - Maximal elements theorems in product FC-spaces and generalized games, J. Math. Anal. Appl. 305 (2005), no. 1, 29-42.

[14] - Nonempty intersection theorems and generalized multi-objective games in product FC-spaces, J. Global Optim. 37 (2007), no. 1, 63-73.

[15] X. P. Ding and J. Y. Park, Fixed points and generalized vector equilibrium problems in generalized convex spaces, Indian J. Pure Appl. Math. 34 (2003), no. 6, 973-990.

[16] Ky Fan, Some properties of convex sets related to fixed point theorems, Math. Ann. 266 (1984), no. 4, 519-537.

[17] F. Giannessi, Theorems of alternative, quadratic programs and complementarity problems, In Variational inequalities and complementarity problems (Proc. Internat. School, Erice, 1978), pp. 151-186, Wiley, Chichester, 1980.

[18] — Vector Variational Inequalities and Vector Equilibria Mathematics Theories, Kiuwer Academic Publishers, London, 2000.

[19] J. Guillerme, Nash equilibrium for set-valued maps, J. Math. Anal. Appl. 187 (1994), no. 3, 705-715.

[20] R. H. He and Y. Zhang, Some maximal elements theorems in FC-spaces, J. Inequal. Appl., doi:10.1155/2009/905605. 
[21] M. Lassonde, Fixed point for Kakutani factorizable multifunctions, J. Math. Anal. Appl. 152 (1990), no. 1, 46-60.

[22] L. J. Lin, Z. T. Yu, and G. Kassay, Existence of equilibria for multivalued mappings and its application to vectorial equilibria, J. Optim. Theory Appl. 114 (2002), no. 1, 189-208.

[23] S. Park and H. Kim, Coincidence theorems on a product of generalized convex spaces and applications to equilibria, J. Korean. Math. Soc. 36 (1999), no. 4, 813-828.

Rong-Hua He

Department of Mathematics

Chengdu University of Information Technology

Chengdu, Sichuan 610103, P. R. China

E-mail address: ywlcd@cuit.edu.cn

Hong-Xu Li

Department of Mathematics

Sichuan University

Chengdu, Sichuan 610064, P. R. China

E-mail address: hoxuli@sohu.com 